\title{
Real-time and accuracy of rapid on-site cytological evaluation of lung cancer
}

\author{
Zhongqin Huang ${ }^{1 \#}$, Dongchun Zhuang ${ }^{2 *}$, Airan Feng ${ }^{2}$, Ling $\mathrm{Ye}^{2}$, Lingling Hong ${ }^{2}$ \\ ${ }^{1}$ Department of Nursing, The Fifth Hospital of Xiamen, Xiamen, Fujian, China; ${ }^{2}$ Department of Respiration, The Fifth Hospital of Xiamen, \\ Xiamen, Fujian, China \\ Contributions: (I) Conception and design: L Hong; (II) Administrative support: L Hong; (III) Provision of study materials or patients: Department \\ of Respiration, The Fifth Hospital of Xiamen; (IV) Collection and assembly of data: Z Huang; (V) Data analysis and interpretation: Z Huang; (VI) \\ Manuscript writing: All authors; (VII) Final approval of manuscript: All authors. \\ "These authors contributed equally to this work. \\ Correspondence to: Lingling Hong. Department of Respiration, The Fifth Hospital of Xiamen, 101 Minan Road, Xiamen, Fujian 361101, China. \\ Email: dazzle-posh@126.com.
}

\begin{abstract}
Background: Although using pathological diagnosis is the gold standard for lung cancer diagnosis, pathological reports take a long time. Rapid on-site assessment (ROSE) is a method to determine whether the quality of the specimens is sufficient for cytopathological diagnosis, which issues a preliminary report during the operation and takes shorter time. The aim of this study is to explore the clinical significance of rapid on-site evaluation (ROSE) in the diagnosis of lung cancer in terms of real-time and accuracy.
\end{abstract}

Methods: A total of 38 suspected lung cancer cases were enrolled from August 2019 to July 2020. Patients received ROSE and pathological examinations at the same time. The coincidence rate of the two diagnostic approaches was calculated, and statistical analysis was carried out to evaluate whether the time difference between the ROSE report and the pathological report reached statistical significance.

Results: A total of 38 suspected lung cancer cases were enrolled from August 2019 to July 2020. Patients received ROSE and pathological examinations at the same time. The coincidence rate of the two diagnostic approaches was calculated, and statistical analysis was carried out to evaluate whether the time difference between the ROSE report and the pathological report reached statistical significance..

Conclusions: ROSE has the advantage of rapid and accurate diagnosis for lung cancer, and has great clinical significance.

Keywords: Rapid on-site evaluation (ROSE); biopsy; pathology; lung cancer

Submitted Oct 19, 2020. Accepted for publication Jan 15, 2021.

doi: $10.21037 /$ tcr-20-3294

View this article at: http://dx.doi.org/10.21037/tcr-20-3294

\section{Introduction}

Lung cancer is the most common cancer in China, followed by gastric cancer. According to an analysis of the prevalence of malignancies in 2015, 787,000 people were diagnosed with lung cancer in China (1). Lung cancer has the highest mortality for both women and men, with a 5 -year survival rate of only $19.7 \%$ (2). In Europe, lung cancer is one of the 3 most common tumor diseases with the highest mortality rate (3). Lung cancer is susceptible to metastasis, resulting in poor prognosis, and the average survival time of patients with metastatic lung cancer is only 3-6 months (4). Pathological diagnosis through various techniques is the gold standard, and includes cytological examination, pleural biopsy, computed tomography (CT), or percutaneous lung biopsy, which provide evidence for diagnosis for lung cancer patients $(5,6)$. Treatment against lung cancer depends on the pathological type, so a clear pathological classification is the prerequisite for treatment. However, it usually takes $2-5$ days for traditional approaches in most domestic 

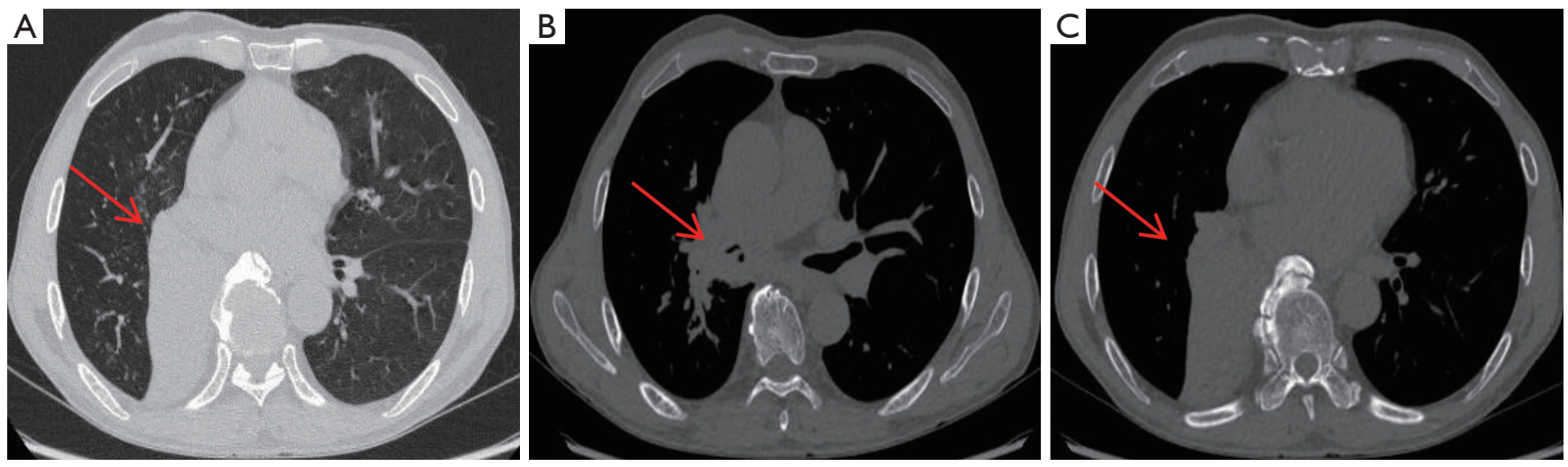

Figure 1 Computed tomography of a 65 -year-old male showing atelectasis caused by bronchial occlusion in the middle and lower lobes of the right lung, and a soft tissue shadow in the right hilum.

hospitals to produce histological results, which not only prolongs the length of hospital stay but also increases financial burden. The frozen section allows rapid diagnosis during surgery, but this technology requires a large amount of specimens. ROSE technology only needs a small amount of specimens for rapid diagnosis, avoiding the risk of bleeding due to excessive clamping of specimens. In addition, preparation process of frozen section is complicated, and such kind of report must be issued by a specialist pathologist. However, pathologists are scarce and the patients have to bear huge economic burden, hardly meeting their demands. Rapid on-site evaluation (ROSE) technology provides an adequate evaluation and preliminary diagnosis in a short time after a partial section is obtained from the target site, printed on glass side, stained, and observed under a microscope $(7,8)$. ROSE is divided into cytological ROSE (C-ROSE) and microbiological ROSE. In the present study, we discuss the clinical significance of C-ROSE for lung cancer. C-ROSE-guided biopsy has been found to increase the positive rate of lung biopsy and can quickly clarify the type of pathology (9). C-ROSE is increasingly applied to clinical practice at home and abroad. However, few studies have compared this technology with traditional pathological report in terms of real-time capability and accuracy. Therefore, the aim of the present study was to discuss the difference between ROSE report and C-ROSE for diagnosing lung cancer. We present the following article in accordance with the MDAR checklist (available at http://dx.doi.org/10.21037/tcr-20-3294).

\section{Methods}

Inclusion criteria include the following: (I) patients who had single or multiple nodules and pleural effusions in the lungs as demonstrated by preoperative enhanced chest CT scan, were immune to the regular anti-infective treatment; (II) patients suspected of lung cancer based on comprehensive analysis of the patient's medical history and auxiliary examination results; (III) patients have completed general preoperative examination to exclude contraindications related to lung biopsy; (IV) patients and family members were told the risk of operation and have signed informed consent before surgery. Exclusion criteria were (I) contraindications to surgery such as coagulation dysfunction, severe heart and lung failure; (II) without complete clinical data. Thirty-eight patients with suspected lung cancer, who had pulmonary space-occupying lesions, were enrolled at the Fifth Hospital of Xiamen from August 2019 to July 2020 (28 males and 10 females, 43-89 years of age, average age: 67.32 years). Of these, 24 were examined by bronchoscopy, 10 by CT-guided percutaneous lung puncture examination, and 4 by pleural biopsy and thoracic closed drainage. When the pathological specimens were sent to the pathology department for routine examination, patients were subjected to ROSE. The results of 2 examinations and the time required for the report were recorded.

\section{Sample collection and detection}

Lesions involving the airway or that are close to the airway were detected by bronchoscopy (Figures 1 and 2). Chest CT and bronchoscope indicated the location of the lesion, followed by bronchoscopic mucosal biopsy and lung biopsy. A disposable $5-\mathrm{mL}$ sterile syringe needle was used to obtain tissue from the biopsy specimens. The tissue was then placed on a slide and smeared to a $1-\mathrm{cm}$ circle tissue block 

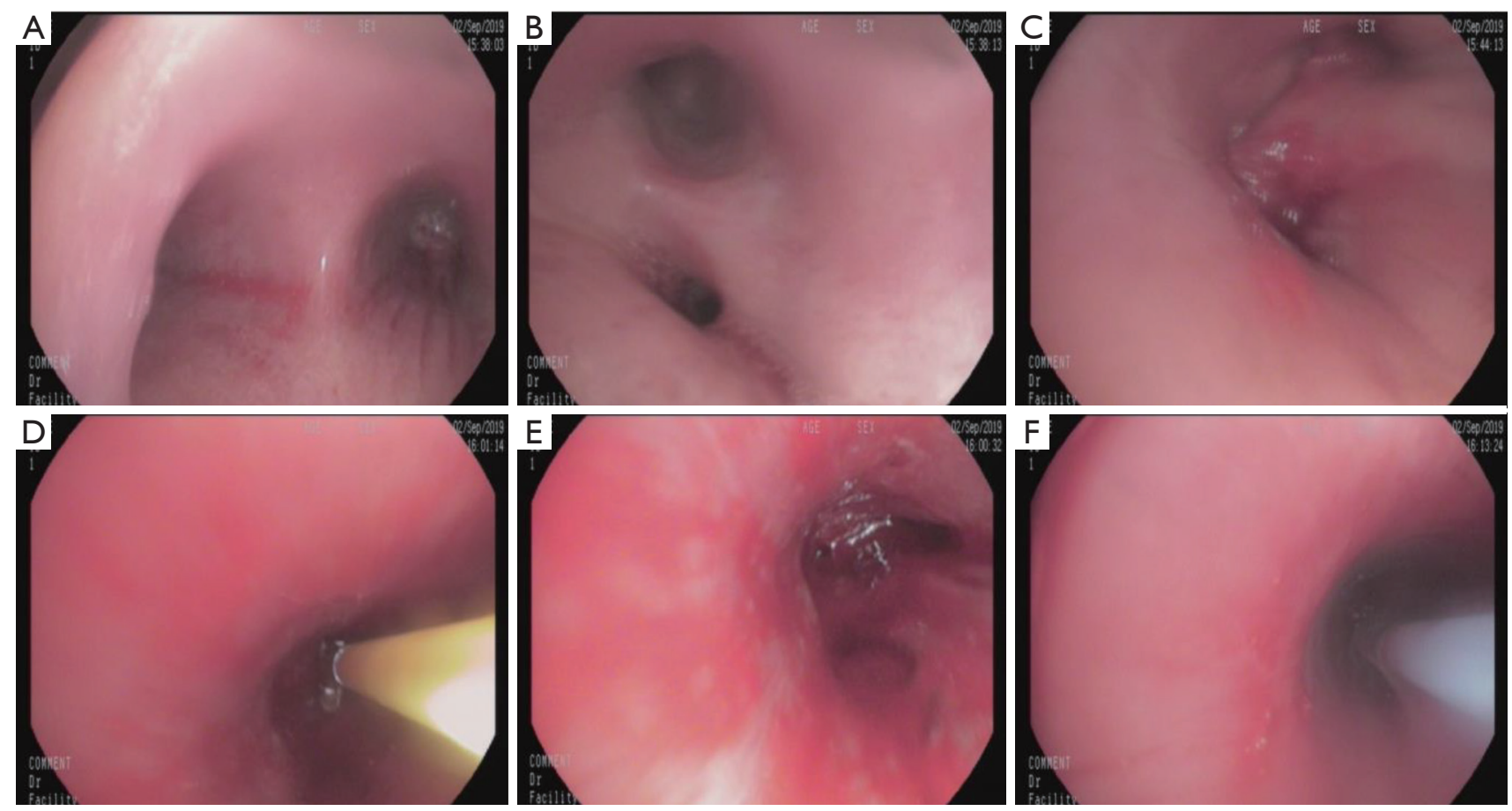

Figure 2 Sixty-five-year-old male patient. (A) Carina, (B) left main bronchus opening, (C) narrow right main bronchus opening, (D) biopsy of right main bronchus mucosa, (E) post-biopsy of right main bronchus mucosa, (F) argon knife for right main bronchus mucosa.

print for ROSE $(10,11)$; the remaining specimens were fixed in $10 \%$ formaldehyde solution and sent to the pathology department for examination. For peripheral lung lesions or lesions that were not easily reached by bronchoscopy, CTguided percutaneous lung biopsy was performed (Figure 3). The puncture needle was inserted into the puncture point indicated by CT to prepare tissue print, and the remaining tissue block was sent to the pathology department. Pleural biopsy and closed pleural drainage were performed for patients with moderate and large pleural effusions (Figure 4). The pleural tissue obtained by the pleural biopsy needle and the drainage pleural effusion were centrifuged to make a ROSE tissue print. The remaining specimens were sent to the pathology department for routine examination.

\section{ROSE technology}

The ROSE technology consists of preparation of section, dyeing, and observation $(10,11)$. After preparing the sections, the sections were immediately subjected to modified Diff-Quick stain (Biotech Development Co., Ltd., Zhuhai, China), immersed in solution A and solution B, and washed with phosphate buffered solution, followed by drying with absorbent paper. The entire staining process could complete within 1-2 min. The sections were then viewed under a microscope for comprehensive analysis. A study published in Chest in 2018 has showed that after 3 months of training in cytopathology knowledge, respiratory physicians also can complete ROSE interpretation, and the accuracy gets close to that of cytopathologists without statistical difference (12). The ROSE report was issued by a respiratory specialist who has been trained in cytopathology If cells presented with tumor cell characteristics in the ROSE roll, they were considered to be a positive specimen. The results guided the subsequent treatment direction.

\section{Pathological techniques}

The tissues were fixed, dehydrated, cleared, and embedded into paraffin. The paraffin was cut into slides and then stained. The slides were then observed by pathologists, and if necessary, immunohistochemistry was conducted.

\section{Ethical statement}

The study was conducted in accordance with the 

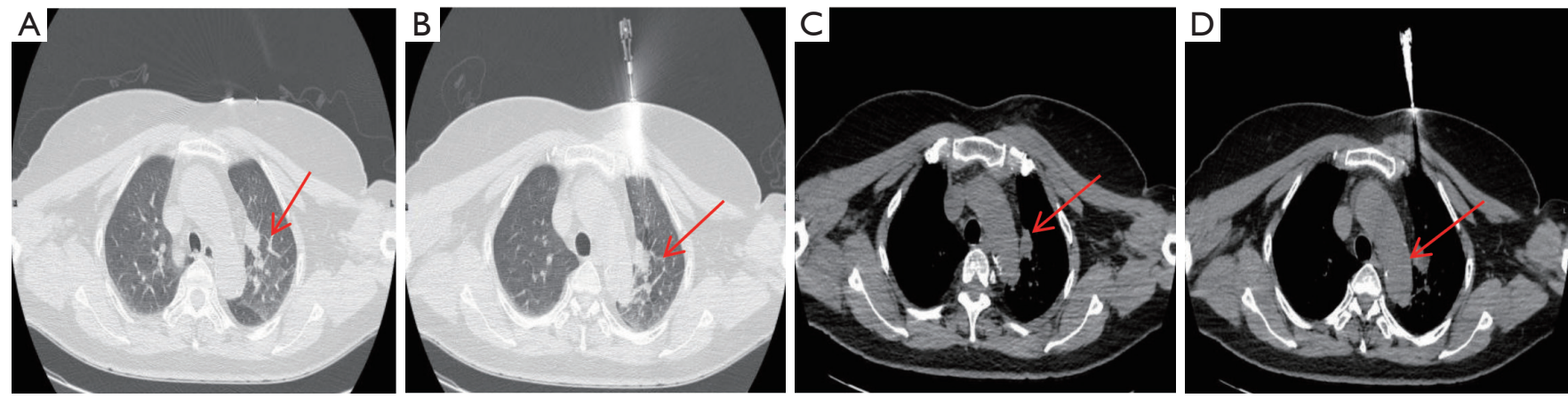

Figure 3 Computed tomography of a 61-year-old female showing soft tissue with a diameter of $0.79 \mathrm{~cm}$ next to the aortic arch of the left upper lobe, followed by percutaneous lung biopsy. Red arrows indicate soft tissue.
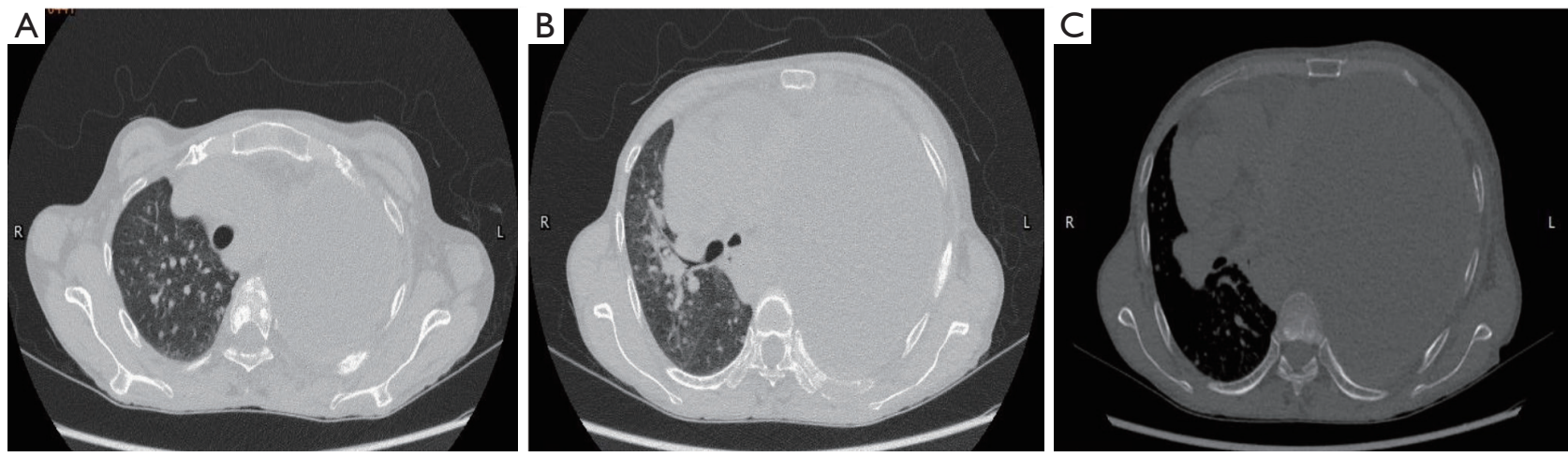

Figure 4 Computed tomography of 44-year-old female showing pleural effusion on the left, followed by pleural biopsy and closed pleural drainage to obtain specimens for examination.

Declaration of Helsinki (as revised in 2013). The study was approved by ethics board of the Fifth Hospital of Xiamen (NO.:2020-XMSDWYY-002) and informed consent was taken from all individual participants.

\section{Statistical analysis}

SPSS version 22.0 (IBM, Armonk, NY, USA) was used for the statistical analysis, and $t$-test was used to compare the count data. $\mathrm{P}<0.05$ was considered statistically significant.

\section{Results}

\section{Results of diagnosis}

The final pathological diagnosis in the 38 patients was lung cancer for all patients, including 21 cases of adenocarcinoma, 11 cases of squamous cell carcinoma, and 6 cases of small cell lung cancer. In the ROSE report, only 1 ROSE report was negative, and the remaining ROSE diagnoses were malignant with complete tumor cell characteristics. Of the 38 patients, 25 were diagnosed by electronic bronchoscopy, 9 by CT-guided lung biopsy, and 4 by pleural biopsy combined with closed thoracic drainage. The diagnosis coincidence of the ROSE technique and pathology was $95.45 \%$ (Table 1).

\section{Comparison of the time between ROSE diagnosis and pathological diagnosis}

After collecting the specimen, we calculated the time of the ROSE report and pathological report. The timing of report was calculated from the first specimen to the production of final report. The ROSE report took 4-52 min to obtain the result, with an average time of $13.84 \mathrm{~min}$, while the pathological report time took $2,832-15,885 \mathrm{~min}$, with an average time of 6,222.05 $\mathrm{min}$ (103.70 h, 4.3 days) (Table 2). The difference between the 2 reports was significant $(\mathrm{P}<0.05)$. 
Table 1 ROSE and pathological diagnosis result

\begin{tabular}{lcccc}
\hline Detection & Positive & Negative & Positive ratio (\%) & Coincidence (\%) \\
\hline ROSE & 37 & 1 & 97.37 & 97.37 \\
Pathology detection & 38 & 0 & 100 & \\
\hline
\end{tabular}

ROSE, rapid on-site evaluation.

Table 2 Comparison between ROSE time and pathological report time

\begin{tabular}{lccc}
\hline Time & $\bar{x} \pm \mathrm{s}(\min )$ & $t$ value & P value \\
\hline ROSE time & $13.84 \pm 9.3$ & -17.79 & $<0.05$ \\
Pathological report time & $6,222.05 \pm 2,151.02$ & & \\
\hline
\end{tabular}

ROSE, rapid on-site evaluation.

\section{Characteristics of lung adenocarcinoma cells under ROSE microscope}

After the tissue specimen print was processed, lung adenocarcinoma cell characteristics were observed under ROSE microscope as follows: (I) large and round cells distributed in piles and clusters; (II) large nucleus, abundant cytoplasm with vacuoles; (III) acinus-, papillary-, and mulberry-like cells; (IV) round nucleus with a cytoplasm; (V) coarse and granular chromatin; and (VI) large and clear nucleolus (Figure 5A). The pathological diagnosis of the same patient was lung adenocarcinoma, as shown in Figure 5 B.

\section{Characteristics of lung squamous cell carcinoma under ROSE microscope}

Lung squamous cell carcinoma cells were observed under ROSE microscope with the following manifestations: (I) irregular cell shape, not round, polygonal, and spindleshaped. Obvious deformity and clear edges; (II) keratinized and uniform plaster-like cytoplasm mainly in red. Some cells with few cytoplasms and even a bare nucleus; (III) nuclear chromatin was densely stained, and the size of the nucleus was irregular and angled with obvious deformities; and (IV) a "positive background" was obvious (Figure 5C). The histopathology of the same patient was finally diagnosed as lung squamous cell carcinoma, as shown in Figure 5D.

\section{Characteristics of small cell lung cancer under ROSE microscope}

ROSE microscope indicated the following characteristics of small cell lung cancer cells: (I) small cancer cells with less cytoplasms or naked nuclei without plasma. Nucleoli were blurred or missing. Nuclear chromatin was granular and unevenly distributed like "ghost faces". Cancer cells were arranged in rows or mosaics, often densely packed; and (II) necrosis and nuclear filaments (Figure 5E). The final diagnosis was small cell lung cancer, as shown in Figure $5 F$.

\section{Discussion}

Diagnostic interventional techniques have good sensitivity and specificity, but there is a risk of trauma in the process of repeated sampling, so the times and scope of punctures should be reduced to ensure sufficient specimens. ROSE technology is widely used in the clinical setting $(9,13,14)$. When combined with biopsy technology, it not only reduces the number of punctures $(15,16)$, but also increases the positive rate of diagnosis, avoid traumas, and reduces risk of complications. After obtaining the sample and after smearing, ROSE is immediately carried out to determine whether the amount of specimens is sufficient and qualified, and a primary diagnosis can be obtained immediately (7). Once a sufficient amount of qualified specimens is obtained, the operation can be terminated immediately, saving time and reducing patient trauma. However, if a sufficient amount of qualified specimens is not obtained, the location and method of sampling can be adjusted to obtain an ideal specimen. Through ROSE test takes longer to obtain samples, it is worthwhile for patients since the eligibility of the puncture specimen by ROSE test can increase the positive rate of pathological results, thereby reducing the risk of secondary puncture. In the present study, the samples of 24 patients were obtained through bronchoscopy. After sampling, the ROSE interpreter immediately prepared, 

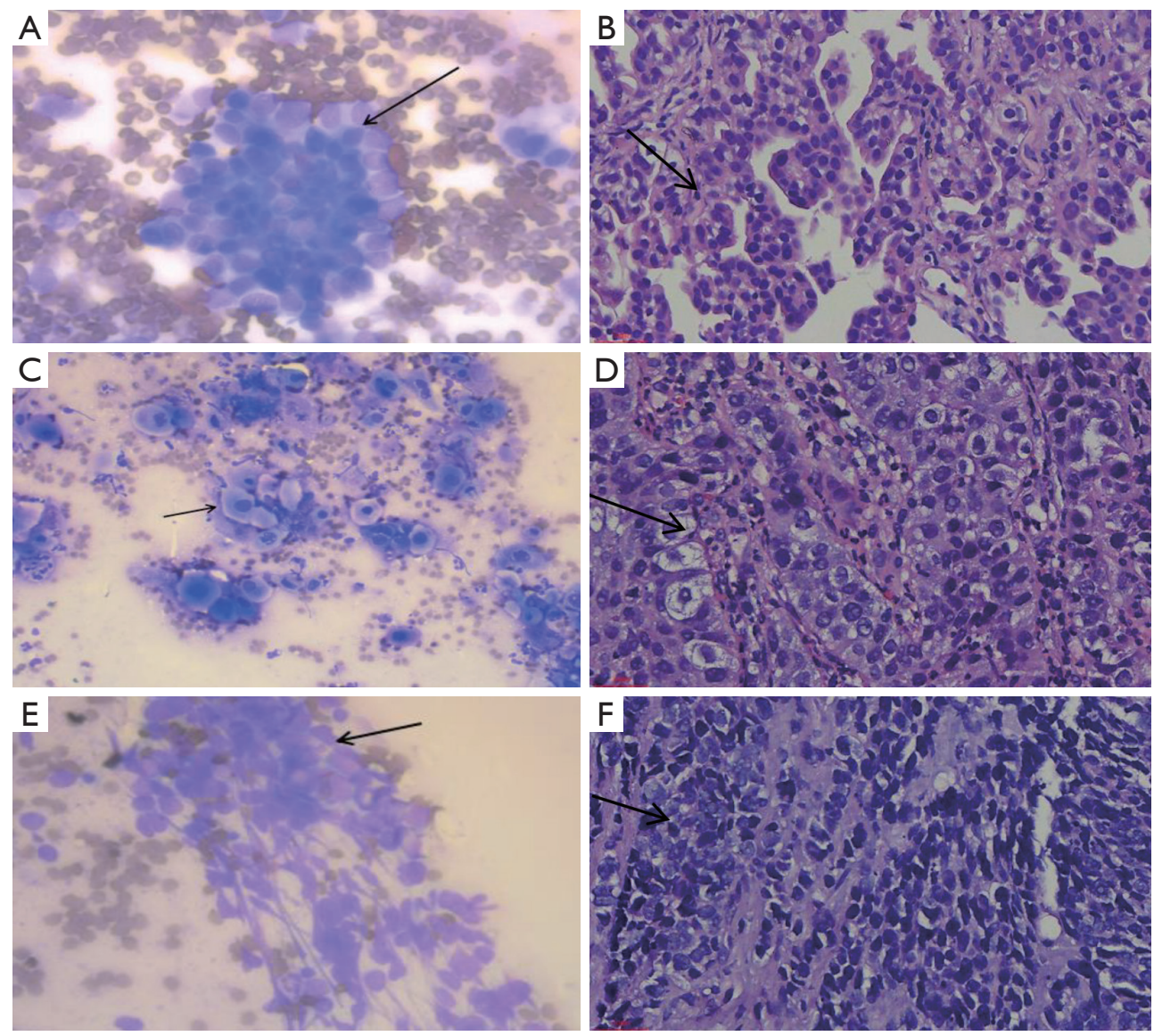

Figure 5 Comparison between rapid on-site evaluation (ROSE) diagnosis and pathological diagnosis. (A) Adenocarcinoma cells of a 61-yearold female patient seen under ROSE microscope $(\times 200)$. Clumped distribution with a large nucleus. Vacuoles are seen in the abundant cytoplasm. (B) Representative pathological images of specimens from a 61-year-old female patient [hematoxylin-eosin staining (HE) $\times 400$ ]. Hyperplastic adenocarcinoma cells are seen under high power lens. (C) Representative ROSE images of lung squamous cell carcinoma under a microscope for a 65 -year-old male patient $(\times 200)$. Irregular cancer cells are seen. (D) Representative pathological images of specimens from a 65 -year-old male patient (HE staining $\times 400$ ). Keratinized squamous cancer cells are seen. (E) Representative ROSE images of small cell lung cancer under a microscope $(\times 200)$, with few cytoplasm, no nucleolus, necrosis, and nuclear filaments for a 44-year-old female patient. (F) Immunohistochemical synaptophysin (+), CD56 (+), and Ki-67 (80\%) for a 44-year-old female patient. Small cell lung cancer is indicated pathologically (HE staining $\times 400)$.

stained, and observed the sections under a microscope. When malignant cells were seen under the microscope and the sample was sufficient, the operation was stopped. While obtaining specimens, we reduced the times of punctures as much as possible. Ten patients underwent CT-guided lung biopsy, as ROSE was performed during the puncture process to increase the positive rate of specimens (17-21). The operation was stopped after the detection of malignant cells. All postoperative pathology results were positive, and the ROSE diagnosis coincided with the pathology report $(97.37 \%)$. The other 4 patients with a large amount of pleural effusion underwent pleural biopsy and closed pleural drainage. Of these patients, malignancies were detected in the pleural tissue print of 3 patients, and 1 patient tested negative. We can increase the number of smears on the same specimen, and secondly improve the technical capabilities of respiratory specialists on-site ROSE, including smearing, staining, and interpretation through specialist training, exchange for further education and learning to further increase the positive rate and coincidence rate of ROSE. Further experiments are required to verify the role of ROSE in pleural tissue. 
While obtaining biopsy tissue, ROSE is often performed routinely to quickly obtain a preliminary diagnosis. Compared with histopathological examination, ROSE is faster and more convenient. ROSE is important for the diagnosis of primary bronchial lung cancer, and some cases can be clearly classified by ROSE technology. In addition, it has been reported that the molecular biologic and genetic technology of cells can be obtained through detecting the ROSE print, such as polymerase chain reaction, fluorescence in situ hybridization (FISH), immunocytochemistry, and second-generation gene sequencing $(22,23)$. Lung cancer has a high incidence and high mortality in China, and most cases are already at advanced stage when diagnosed (24). The main treatment opinions for lung cancer include surgery, chemotherapy, targeted therapy, and immunotherapy. Treatment methods are mainly based on pathological diagnosis and genetic test results. The demand for targeted therapy and immunotherapy is increasing, but these therapies depend on genetic testing, and there must be an accurate diagnosis to receive specific therapies. In the early stage of the disease, when the lesion is small, tumor cells can be reflected in ROSE report, and when the pathological specimen report is negative, the ROSE print can be used for staining to confirm the diagnosis and to guide treatment $(25,26)$. In the present study, the average ROSE time was about $13.84 \mathrm{~min}$, and the average pathology report time was $6,222.05 \mathrm{~min}$ (about 4.3 days), which significantly longer than the ROSE time. In the absence of ROSE, the possibility of negative results and repeated examinations increases, further extending the diagnosis time, increasing hospitalization costs, and causing extra trauma for the patient.

During the diagnosis of lung cancer, the application of ROSE technology can make up for the deficiency of puncture or biopsy and pathological reports, as ROSE immediately provides a primary diagnosis with higher accuracy, and only takes about $13.84 \mathrm{~min}$.

\section{Acknowledgments}

Funding: The present study was supported by the Xiamen Science and Technology Project (grant no. 3502Z20194089) and the Science and Technology Guiding Project of Fujian Provincial (grant no. 2020D024).

\section{Footnote}

Reporting Checklist: The authors have completed the MDAR reporting checklist. Available at http://dx.doi.org/10.21037/ tcr-20-3294

Data Sharing Statement: Available at http://dx.doi. org/10.21037/tcr-20-3294

Conflicts of Interest: All authors have completed the ICMJE uniform disclosure form (available at http://dx.doi. org/10.21037/tcr-20-3294). The authors have no conflicts of interest to declare.

Ethical Statement: The authors are accountable for all aspects of the work in ensuring that questions related to the accuracy or integrity of any part of the work are appropriately investigated and resolved. The study was conducted in accordance with the Declaration of Helsinki (as revised in 2013). The study was approved by ethics board of the Fifth Hospital of Xiamen (NO.:2020-XMSDWYY-002) and informed consent was taken from all individual participants.

Open Access Statement: This is an Open Access article distributed in accordance with the Creative Commons Attribution-NonCommercial-NoDerivs 4.0 International License (CC BY-NC-ND 4.0), which permits the noncommercial replication and distribution of the article with the strict proviso that no changes or edits are made and the original work is properly cited (including links to both the formal publication through the relevant DOI and the license). See: https://creativecommons.org/licenses/by-nc-nd/4.0/.

\section{References}

1. Zheng RS, Sun KX, Zhang SW, et al. Zhonghua Zhong Liu Za Zhi 2019;41:19-28.

2. Zeng H, Chen $\mathrm{W}$, Zheng R, et al. Changing cancer survival in China during 2003-15: a pooled analysis of 17 population-based cancer registries. Lancet Global Health 2018;6:e555-67.

3. Ferlay J, Colombet M, Soerjomataram I, et al. Cancer incidence and mortality patterns in Europe: Estimates for 40 countries and 25 major cancers in 2018. Eur J Cancer 2018;103:356-87.

4. Ettinger DS, Wood DE, Aisner DL, et al. Non-Small Cell Lung Cancer, Version 5.2017, NCCN Clinical Practice Guidelines in Oncology. J Natl Compr Canc Netw 2017;15:504-35.

5. National Lung Screening Trial Research Team. Lung 
Cancer Incidence and Mortality with Extended Followup in the National Lung Screening Trial. J Thorac Oncol 2019;14:1732-42.

6. Lerner AD, Feller-Kopman D. Bronchoscopic Techniques Used in the Diagnosis and Staging of Lung Cancer. J Natl Compr Canc Netw 2017;15:640-7.

7. Wohlschläger J, Darwiche K, Ting S, et al. Rapid on-site evaluation (ROSE) in cytological diagnostics of pulmonary and mediastinal diseases. Pathologe 2012;33:308-15.

8. Nakajima T, Yasufuku K, Saegusa F, et al. Rapid on-site cytologic evaluation during endobronchial ultrasoundguided transbronchial needle aspiration for nodal staging in patients with lung cancer. Ann Thorac Surg 2013;95:1695-9

9. Wang J, Zhao Y, Chen Q, et al. Diagnostic value of rapid on-site evaluation during transbronchial biopsy for peripheral lung cancer. Jpn J Clin Oncol 2019;49:501-5.

10. Collins BT, Chen AC, Wang JF, et al. Improved laboratory resource utilization and patient care with the use of rapid on-site evaluation for endobronchial ultrasound fine-needle aspiration biopsy. Cancer Cytopathol 2013;121:544-51.

11. Guo H, Liu S, Guo J, et al. Rapid on-site evaluation during endo-bronchial ultrasound-guided transbronchial needle aspiration for the diagnosis of hilar and mediastinal lymphadenopathy in patients with lung cancer. Cancer Lett 2016;371:182-6.

12. Hopkins E, Moffat D, Smith C, et al. Accuracy of rapid on-site evaluation of endobronchial ultrasound guided transbronchial needle aspirates by respiratory registrars in training and medical scientists compared to specialist pathologists-an initial pilot study. J Thorac Dis 2018;10:3922-7.

13. Medina Chamorro FM, Calle JA, Stein JE, et al. Experience of the Implementation of Rapid On-Site Evaluation in Ultrasound-Guided Fine-Needle Aspiration Biopsy of Thyroid Nodules. Curr Probl Diagn Radiol 2018;47:220-4.

14. Iglesias-Garcia J, Lariño-Noia J, Abdulkader I, et al. Rapid on-site evaluation of endoscopic-ultrasound-guided fineneedle aspiration diagnosis of pancreatic masses. World J Gastroenterol 2014;20:9451-7.

15. Oki M, Saka H, Kitagawa C, et al. Rapid on-site cytologic evaluation during endobronchial ultrasound-guided transbronchial needle aspiration for diagnosing lung cancer: a randomized study. Respiration 2013;85:486-92.

16. Madan NK, Madan K, Jain D, et al. Utility of conventional transbronchial needle aspiration with rapid on-site evaluation (c-TBNA-ROSE) at a tertiary care center with endobronchial ultrasound (EBUS) facility. J Cytol 2016;33:22-6

17. Bandyopadhyay A, Laha R, Das TK, et al. CT guided fine needle aspiration cytology of thoracic mass lesions: a prospective study of immediate cytological evaluation. Indian J Pathol Microbiol 2007;50:51-5.

18. Di Donna A, Bazzocchi M, Dolcet F, et al. CTguided transthoracic needle aspiration of solitary lung lesions. Personal experience in 118 cases. Radiol Med 1995;89:287-94.

19. Mukherjee S, Bandyopadhyay G, Bhattacharya A, et al. Computed tomography-guided fine needle aspiration cytology of solitary pulmonary nodules suspected to be bronchogenic carcinoma: Experience of a general hospital. J Cytol 2010;27:8-11.

20. Anila KR, Nayak N, Venugopal M, et al. Role of Rapid On-site Evaluation in CT-guided Fine Needle Aspiration Cytology of Lung Nodules. J Cytol 2018;35:229-32.

21. Fassina A, Corradin M, Zardo D, et al. Role and accuracy of rapid on-site evaluation of CT-guided fine needle aspiration cytology of lung nodules. Cytopathology 2011;22:306-12.

22. Chandra S, Chandra H, Sindhwani G. Role of rapid onsite evaluation with cyto-histopathological correlation in diagnosis of lung lesion. J Cytol 2014;31:189-93.

23. Rekhtman N, Brandt SM, Sigel CS, et al. Suitability of thoracic cytology for new therapeutic paradigms in non-small cell lung carcinoma: high accuracy of tumor subtyping and feasibility of EGFR and KRAS molecular testing. J Thorac Oncol 2011;6:451-8.

24. Siegel R, Desantis C, Jemal A. Colorectal cancer statistics, 2014. CA Cancer J Clin 2014;64:104-17.

25. Jain D, Ramachandrappa VS, Singh V, et al. Use of Exfoliative Specimens and Fine-Needle Aspiration Smears for Mutation Testing in Lung Adenocarcinoma. Acta Cytol 2017;61:455-61.

26. Billah S, Stewart J, Staerkel G, Chen S, Gong Y, Guo M. EGFR and KRAS mutations in lung carcinoma: molecular testing by using cytology specimens. Cancer Cytopathol 2011;119:111-7.

(English Language Editor: R. Scott)

Cite this article as: Huang Z, Zhuang D, Feng A, Ling Y, Hong L. Real-time and accuracy of rapid on-site cytological evaluation of lung cancer. Transl Cancer Res 2021;10(1):479486. doi: $10.21037 /$ tcr-20-3294 